\title{
Reduction in the level of antibodies against heat shock proteins 60 during different hormonal protocols in postmenopausal women
}

\author{
Agnieszka Rajtar-Ciosek, Olga Kacalska-Janssen, Andrzej Zmaczyński, Jakub Wyroba, Rita Tomczyk, \\ Joanna Wiatr, Anna Gałuszka-Bednarczyk, Tomasz Bereza, Tomasz Milewicz, Józef Krzysiek
}

Department of Endocrynological Gynecology, Medical College, Jagiellonian University, Krakow, Poland

\begin{abstract}
Introduction: In current literature, the immune-inflammatory theory of atherosclerosis is widely discussed. The role of how heat shock proteins 60 (HSP60) lead to the development of the atheromatous plaque is especially underlined. The aim of the study is to estimate the influence of three hormonal protocols on behavior of antibodies against HSP60. It determines the state of endothelium in postmenopausal women.

Material and methods: The study was carried out on 90 women between 2007 and 2012. All the women were in their menopausal age ( $51 \pm 3$ years), from the south region of Poland, with a follicle stimulating hormone (FSH) level above $25 \mathrm{mIU} / \mathrm{ml}$, and with menopausal symptoms disturbing their normal daily activity. The study was done for a period of 6 months. Three groups of 30 randomized patients were formed. In the first group we used transdermal estrogen therapy in a $37.5 \mu \mathrm{g} / 24 \mathrm{~h}$ dose combined with a $10 \mathrm{mg}$ dose of dydrogesterone. In the second group we applied transdermal estrogen therapy in a $50 \mu \mathrm{g} / 24 \mathrm{~h}$ dose with $2.5 \mathrm{mg}$ of oral medroxyprogesterone. In both these groups, gestagens were administered continuously. In the third group, we prescribed continuous, oral, low-dose combined estrogen-gestagen therapy with $1 \mathrm{mg}$ of ethinyl estradiol and $0.5 \mathrm{mg}$ of norethisterone acetate. The control group consisted of 30 volunteers who were also from the south region of Poland, in good health, with menopausal symptoms, no menstrual period for the last 12 months, selected considering their age and weight, with an FSH level above $25 \mathrm{mIU} / \mathrm{ml}$ and with normal levels of thyroid stimulating hormone (TSH) and prolactin. All patients treated and in the control group were seronegative to Chlamydia pneumonia for the entire duration of the study. In the analysis conducted, nonparametric tests were used (Mann-Whitney $U$ test, Wilcoxon test, Kruskal-Wallis test - ANOVA).

Results: After 6 months of hormonal therapy, we found that all schemes of treatment promote a significant reduction in antibodies against HSP60 in all treated groups vs. the control group.

Conclusions: All of the investigated estrogen protocols have a favorable impact on the blood level of HSP60 antibodies in early postmenopausal women who have no cardiovascular risk factors. It triggers a better condition of endothelium.
\end{abstract}

Key words: heat shock protein 60 (HSP60), estrogen therapy.

\section{Introduction}

There are many inflammatory-immune markers which play an important prognostic role in modern medicine, particularly in peri- and postmenopausal time. An especially interesting issue is the possible impact of postmenopausal lack of sex hormone production and replacement therapy on a vessel's immuneinflammatory condition [1]. Increased levels of those markers are signs of a chronic immune-inflammatory process in atherosclerosis, arterial hypertension, obesity, diabetes mellitus, and others [2].

In current literature, the immune-inflammatory theory of atherosclerosis is widely discussed. Heat shock proteins 60 (HSP60) and their role in the development of the atheromatous plaque are especially underlined.
They are intracellular molecules and signaling intercellular proteins, and have the ability to initiate cytokine secretion and expression of cells' adhesion molecules. The production of heat shock proteins (also called 'stress proteins') is stimulated by a sudden increase in intracellular temperature, and by any other kind of cellular stress such as oxidative stress, viral infection, exposure to some chemical substances (e.g. ethanol), cytokines, and heavy metal ions; which all lead to the dysfunction of endothelial cells. The presence of human native HSP60 on a vessel's endothelial surface might lead to an autoimmune reaction, depending on antibody or complement cytotoxic reactions [2].

The exposure of the host's HSP60 on the endothelial cells and macrophage surfaces causes these cells to be damaged by anti-HSP60 immunoglobulins [2]. 
Antibodies of HSP60 are present in small concentration in the blood of healthy individuals; the physiological reaction remains unclear [3].

It has been proven that monoclonal antibodies specific for HSP60 and antibodies against the HSP60 - a family isolated from human serum have a cytotoxic impact on the endothelium [2-6]. These studies demonstrate that antibodies against HSP60 might play a role in vessel damage and atherosclerosis etiopathogenesis [2-6].

HSP60 expression might also be secondary to the inflammatory process when the cytokine expression in the endothelium and in leukocytes leads to a HSP expression in the vessel wall. HSP60 were once considered as intracellular molecules, but these proteins can be released from live cells to the extracellular space through a mechanism which is still unclear [2-4]. Extracellular HSP60 are signaling molecules and influence several inflammatory reactions.

These proteins might have an impact on atherosclerosis development acting as target autoantigens for reactive T cells that damage their own HSP60 [2].

Having knowledge about the beneficial influence of estrogens on healthy endothelial cells as well as the inflammatory theory of atherosclerosis, we carried out the study aimed to illustrate the influence of three different forms of hormonal protocols on the behavior of antibodies against HSP60 in healthy postmenopausal women with climacteric symptoms, and compared these results to the control group.

\section{Material and methods}

\section{Series description, inclusion criteria}

The study was carried out on 90 women hospitalized or treated in the outpatient clinic at the Endocrinological Gynecology Department, Jagiellonian University between 2007 and 2012. All the patients were in menopausal age (51 \pm 3 years), from the south region of Poland, with follicle stimulating hormone (FSH) levels above $25 \mathrm{mIU} / \mathrm{ml}$, with menopausal symptoms disturbing their normal daily activity [7]; they all fulfilled criteria for hormonal therapy specified by the Standards of Polish Gynecological Society. The study was done for a period of 6 months, during which there were no lifestyle changes, only observation of whether or not quality of life improved (measured by the Green Scale). No other drugs due to chronic illnesses were taken. Three groups of 30 randomized patients were formed. The study was a double-blind randomized trial, the patients were selected to a proper group by the computer. None of the doctors were involved in the study. In the first group we gave transdermal estrogen therapy in a $37.5 \mu \mathrm{g} / 24 \mathrm{~h}$ dose combined with a $10 \mathrm{mg}$ dose of dydrogesterone taken orally. In the second group we prescribed transdermal estrogen therapy in a $50 \mu \mathrm{g} / 24 \mathrm{~h}$ dose with $2.5 \mathrm{mg}$ of oral medroxyprogesterone. In both these groups gestagens were administered continuously. In the third group we applied a continuous, oral, low-dose combined estrogen-gestagen therapy with $1 \mathrm{mg}$ of ethinyl estradiol and $0.5 \mathrm{mg}$ of norethisterone acetate.

Group I $(n=30): 37.5 \mu \mathrm{g} / 24 \mathrm{~h}$ of transdermal $17 \beta$-estradiol, in combination with $10 \mathrm{mg}$ of dydrogesterone (commercial products: Oesclim 37.5 and Duphaston - tablets), abbreviation: 37.5+D.

Group II $(n=30): 50 \mu g / 24 \mathrm{~h}$ of transdermal $17 \beta$-estradiol, in combination with $2.5 \mathrm{mg}$ of medroxyprogesterone (commercial products: Oesclim 50 and Provera $5 \mathrm{mg}$ - half a tablet), abbreviation: 50+MPA.

Group III $(n=30)$ : $1 \mathrm{mg}$ of $17 \beta$-estradiol in combination with $0.5 \mathrm{mg}$ norethisterone acetate as a continuous oral therapy (commercial product: Activelle), abbreviation: Activelle.

Selection of these therapy schemes was suitable. Cho's in-vivo data have shown that oral gestagen therapy has the ability to relax smooth muscle cells by slackening calcium channels, independent of dose [8]. Thirty volunteers also from the south region of Poland, in a good health condition, without chronic diseases, with menopausal symptoms, with no menstrual period in the last 12 months, formed the control group. All patients treated and in the control group were seronegative to Chlamydia pneumonia for the entire duration of the experiment.

\section{Exclusion criteria}

In our study, candidates with the following medical records were excluded: taking hormonal menopausal therapy at the time of evaluation (medical history), suffering from unexplained uterine bleeding (medical history), with a history of endometriosis (medical history, transvaginal ultrasound [USG]), with uterine myoma(s) (medical history, transvaginal USG), with arterial hypertension (daily blood pressure records - systolic pressure less than $140 \mathrm{~mm} \mathrm{Hg}$ and diastolic pressure less than $90 \mathrm{~mm} \mathrm{Hg}$ were accepted as normal), with coronary artery disease (echocardiogram, electrocardiography, cardiac stress test), with diabetes mellitus (oral glucose tolerance test with 75 g of glucose), with an active or chronic inflammatory process (medical history, C-reactive protein $>10 \mathrm{ng} / \mathrm{dl}$, white blood cells $[\mathrm{WBC}]>10000 / \mathrm{mm}^{3}$ ), with autoimmune diseases (medical history), with a liver dysfunction (liver function blood tests), with kidney diseases (sodium, potassium, creatinine or blood urea level beyond the norm), with any neoplasms in their medical history, who have undergone thrombophlebitis or venous thromboembolism (medical history), with cholelithiasis, but not after cholecystectomy (medical history), with seizures in the course of a disease including epilepsy (medical history), with migraine (medical 
history), overweight or obese (body mass index - BMI - score equal to or more than 25 and with the waist-hip ratio equal to or more than 0.78 ).

Smoking and alcohol consumption were taken into consideration in medical history as well.

The following tests were performed for each patient:

- detailed medical history considering arterial hypertension, diabetes mellitus, smoking, inflammatory conditions, liver and kidney diseases, neoplasms, and present pharmacological treatment,

- physical examination with hemodynamic evaluation,

- blood pressure measurement,

- anthropometric measurements including body weight and height (BMI), and waist and hipline (waist-hip ratio - WHR),

- biochemical tests including morphology, hormone levels (FSH, E2 - estradiol, TSH - thyroid stimulating hormone, PRL - prolactin), total cholesterol, LDL-cholesterol, HDL-cholesterol, and triglycerides,

- antibodies against HSP60 - ELISA immunoenzymatic method,

- IgA antibodies against Chlamydia pneumonia.

All patients were informed about the medical study and joined it voluntarily, giving their written consent. Our study has been approved by the Ethics Committee of the Jagiellonian University.

Serum samples were collected and stored at $-70^{\circ} \mathrm{C}$. All samples were tested at the same time (May 2011). All hormones were determined by immunoassay "ECLIA". Total cholesterol and high density lipoprotein (HDL) levels were determined by enzymatic and colorimetric methods. Plasma triglyceride levels were determined with an enzymatic commercial kit (Abbot). Low density lipoprotein (LDL) levels were estimated according to the Friedewald equation. Antibodies against HSP60 were determined by an immunoenzymatic method using a commercial kit (Stressgen Biotechnologies). The normal level for HSP60 antibodies: 7.81-250 ng/ml, and sensitivity $3 \mathrm{ng} / \mathrm{ml}$. IgA antibodies against Chla- mydia pneumoniae were also examined using the immunoenzymatic method (ANI Labsystem, Finland).

\section{Statistical analysis}

In the analysis conducted, nonparametric tests were used.

Analyzed parameters in treated groups were compared using the Mann-Whitney $U$ test. By using the Wilcoxon test, the obtained results in examination 1 (before the treatment) and examination 2 (six months after the hormonal therapy was initiated) in each study group were compared. The differences in outcomes of investigations in resolved materials were confronted using Kruskal-Wallis test (ANOVA), and in cases where significant changes were shown between groups, a multiple comparison test was applied to find those differences. The Pearson's linear correlation coefficient was used to estimate correlations between analyzed parameters and between changes in these factors. $p<0.05$ was statistically significant. Lack of statistical significance was indicated by the abbreviation NS (nonstatistically significant).

Results were shown as a table and figure. Calculations and analysis were made using STATISTICA 7.1.

\section{Results \\ Characteristics of the study groups}

Women in our study groups do not differ significantly regarding age, anthropometric measurements, blood levels of hormones (estradiol, FSH, PRL, and TSH), total cholesterol, LDL and HDL cholesterol and triglycerides. There was no statistically significant difference in the blood level of anti-HSP60 antibodies in the study groups. The results for the 90 participants in the study and control groups are shown in Table I and Figure 1.

Tab. I. Levels of antibodies against heat shock proteins 60 (HSP60) in examination 1 (before therapy) and examination 2 (six months after starting hormonal therapy) in examined groups

\begin{tabular}{|c|c|c|c|c|c|c|c|}
\hline \multirow[t]{2}{*}{ Group } & \multicolumn{6}{|c|}{ Antibodies HSP60 } & \multirow[t]{2}{*}{$p$} \\
\hline & Exam. & $\mathbf{x}$ & SD & Min & $\mathrm{Me}$ & Max & \\
\hline \multirow[t]{2}{*}{ Activelle } & 1 & 279365 & 204350 & 40881 & 145838 & 789986 & 0.01 \\
\hline & 2 & 148152 & 111914 & 41562 & 68340 & 468195 & \\
\hline \multirow[t]{2}{*}{ 50+MPA } & 1 & 122616 & 87398 & 27719 & 93076 & 265091 & 0.05 \\
\hline & 2 & 80149 & 80038 & 22954 & 104990 & 404881 & \\
\hline \multirow[t]{2}{*}{$37.5+D$} & 1 & 150094 & 200356 & 32587 & 75716 & 690338 & 0.003 \\
\hline & 2 & 100658 & 167648 & 27946 & 80822 & 684462 & \\
\hline \multirow[t]{2}{*}{ Control } & 1 & 92849 & 83744 & 21592 & 65731 & 260552 & 0.002 \\
\hline & 2 & 165484 & 62534 & 26811 & 71290 & 282791 & \\
\hline
\end{tabular}


The results demonstrated that after 6 months, hormonal therapy promoted a significant reduction in antibodies against HSP60 in all treated groups vs. the control group, where we observed an increase in the examined antibodies (group I $p=0.003$, group $\| p=0.05$, group III $p=0.01$, vs. control group $p=0.002$, using paired Wilcoxon test) (Table I).

Kruskal-Wallis test (ANOVA) (Fig. 1) showed statistically significant differences ( $p=0.001$ ) between changes in the control group and changes in all study groups. To localize these differences, a multiple comparison test was performed, which did not detect any statistically significant changes. Clinically, regardless of the dose of estrogen, way of its administration, kind of oral gestagen, they all have a positive impact on antibodies against HSP60.

\section{Discussion}

Heat shock proteins belong to a group of conservative proteins which are produced by almost every organism. These proteins are produced in the human body, and in microorganisms such as: Chlamydia pneumoniae, and mycobacterium. In physiological conditions of the body, HSP are present mainly in mitochondria, where they take part in the stabilization of internal proteins.

An interesting observation was presented by Veres et al. and Burian et al. who noted that although there is a 75\% homology between mycobacterial HSP65 and human HSP60, levels of their antibodies are only weakly correlated. The immunological response to human heat shock proteins should therefore be further explored $[9,10]$.

Our original study is one of the few papers investigating the influence of different forms of hormonal therapy on the behavior of antibodies against HSP60 in healthy postmenopausal women. The results presented are different from observations by Uint et al. [11] who showed an increased level of antibodies against HSP60 vs. the control group in women with a mean age of 62 , using conjugated estrogens in 0.625 mg doses with medroxyprogesterone acetate in a 2.5 $\mathrm{mg}$ dose, administered continuously. It is worth underlining that the women in the examined population were different, which probably had an influence on the results.

In our study we proved that there is a beneficial effect on HSP60 after hormonal treatment comparing to the control group. However, we would like to emphasize that there is no statistically significant difference between the discussed groups based on the dose and way of administration of estrogens, or the type of gestagen given.

In our own data, a linear correlation was shown between changes of antibodies against HSP60 and changes of BMI $(r=0.3330, p=0.036)$. An inversely proportional correlation between changes of antibodies

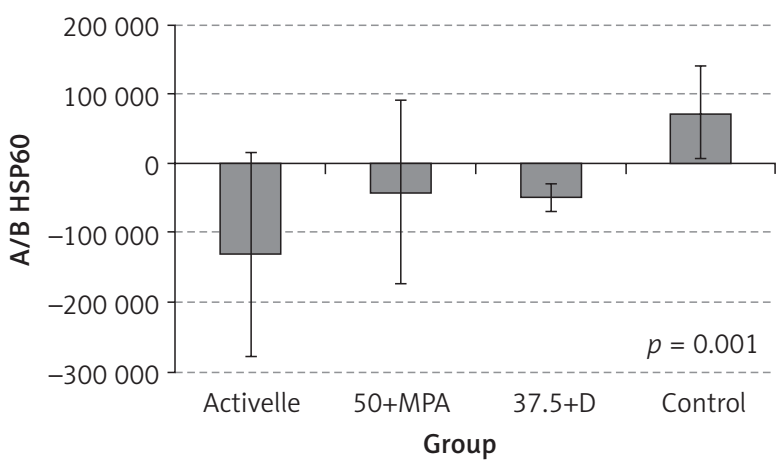

Fig. 1. The comparison of changes in levels of antibodies against heat shock proteins 60 (HSP60) between examined groups

against HSP60 and changes of the estradiol level was also detected $(r=-0.2653, p=0.048)$.

In a paper written by $\mathrm{Yu}$ et al., the positive effects of estradiol (E2) on the cardiac function are mediated via $\beta$-receptor of estradiol and its receptor induces upregulation of heat shock proteins. It plays a significant role in the E2-mediated cardioprotection after trauma haemorrhage in Sprague-Dawley rats [12].

\section{Conclusions}

In conclusion, any of the investigated estrogen protocols has a favorable impact on the blood level of HSP60 antibodies in postmenopausal women with no cardiovascular risk factors.

The essential element in the prevention of endothelial dysfunction is to search for new biochemical or immunological markers with a prognostic value; HSP60 being one of them. This heat shock protein is a marker of the formation and progression of the endothelial dysfunction, but its clinical role warrants further investigations.

\section{Disclosure}

Authors report no conflicts of interest.

\section{References}

1. Rossouw JE, Anderson GL, Prentice RL, et al. Risks and benefits of estrogen and progestin in healthy postmenopausal women: principal results from the Women's Health Initiative randomized controlled trial. JAMA 2002; 288: 321-333.

2. Dembińska-Kieć A, Partyka Ł, Polus A. Miażdżyca procesem autoimmunologicznym? Przegl Lek 2001; 58: 12.

3. Falck G. Acute and persistent Chlamydia pneumoniae infections in Swedish primary health care. Acta Universitatis Upsaliensis, Uppsala 1996.

4. Fryer RH, Schwobe EP, Woods ML, et al. Chlamydia species infect human vascular endothelial cell and induce procoagulant activity. J Investig Med 1997; 45: 168-174.

5. Grayston JT, Kuo CC, Coulson AS, et al. Chlamydia pneumoniae (TWAR) in atherosclerosis of the carotid artery. Circulation 1995; 92: 3397-3400.

6. Grayston JT, Mandell GL, Bennett JE, et al. Chlamydia pneumoniae (TWAR). In: Mandell, Douglas and Bennett's Principles and Practice of Infectious Diseases. Churchill Livingstone, New York 1995. 
7. Wielgoś M, Mazanowska N, Pietrzak B. Atrophic vaginitis: symptoms, diagnosis and treatment. Prz Menopauzalny 2013; 17: 434-437.

8. Cho SH. The vascular effects of progesterone/progestogens. The Menopause the Millennium. Parthenon Publishing, London 2000; 47: 293297.

9. Veres A, Fust G, Smieja M, et al. Heart Outcomes Prevention Evaluation (HOPE) Study Investigators: Realationship of anti-60 kDa heat shock protein and ant-cholesterol antibodies to cardiovascular events. Circulation 2002; 106: 2775-2780.

10. Burian K, Kis Z, Virok D, et al. Independent and joint effects of antibodies to human heat-shock protein 60 and Chlamydia pneumoniae infec- tion in the development of coronary atherosclerosis. Circulation 2001; 103: 1503-1508.

11. Uint L, Gebara OC, Pinto LB, et al. Hormone replacement therapy increases levels of antibodies against heat shock protein 65 and certain species of oxidized low density lipoprotein. Braz J Med Biol Res 2003; 36: 491-494.

12. Yu HP, Shimizu T, Choudhry MA, et al. Mechanism of cardioprotection following trauma-hemorrhagic shock by a selective estrogen receptorbeta agonist: up-regulation of cardiac heat shock factor-1 and heat shock proteins. J Mol Cell Cardiol 2006; 40: 185-194. 